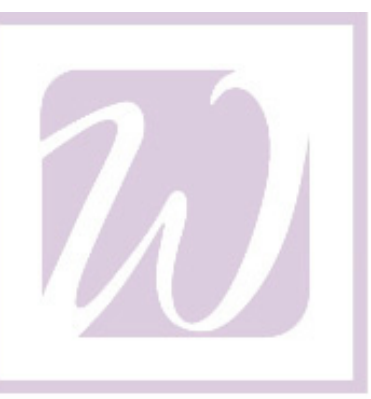

UW-WHITEWATER

Searching for Nonlinearities in Real Exchange Rates

By

Yamin Ahmad

Stuart Glosser

Working Paper 09 - 01

University of Wisconsin - Whitewater

Department of Economics

$4^{\text {th }}$ Floor Carlson Hall

800 W. Main Street

Whitewater, WI 53538

Tel: (262) $472-1361$ 


\title{
Searching for Nonlinearities in Real Exchange Rates
}

\author{
Yamin Ahmad ${ }^{\dagger}$ and Stuart Glosser \\ University of Wisconsin - Whitewater
}

\begin{abstract}
A recent innovation in modeling exchange rates has been the use of nonlinear techniques such as threshold autoregressive models and its smooth transition variants. This paper investigates the smooth transition autoregressive (STAR) modeling strategy in an application to real exchange rates. The key findings are as follows. First, using the methodology advocated by Teräsvirta (1994), we find evidence of nonlinear dynamics for several of the spot dollar real exchange rates using monthly data on five of the G7 countries. However, once estimated, we find that the STAR specification is appropriate for only one of the three exchange rate series indicated to be an ESTAR process. Moreover, using simulations, we show that the underlying methodology used to detect nonlinearities in the data exhibit substantial size biases, which we attribute to influential observations. We also investigate an alternative nonlinear specification and find that we can model the dollar-sterling and the dollar-lira real exchange rates better as an open-loop TAR process instead of a SETAR process.
\end{abstract}

JEL Classification: F30

Keywords: Nonlinear models of exchange rates, threshold models, ESTAR, TAR, PPP.

Current Version: November 21 ${ }^{\text {st }}, 2007$

\footnotetext{
† Corresponding Author: Department of Economics, University of Wisconsin Whitewater, 800 W Main Street, Whitewater, WI 53190 Email: ahmady@uww.edu, Homepage: http://facstaff.uww.edu/ahmady/ Tel: (262) 4725576

$\ddagger$ Department of Economics, University of Wisconsin Whitewater, 800 W Main Street, Whitewater, WI 53190

Email: glossers@uww.edu, Tel: (262) 4725580
} 


\section{Introduction}

The literature on real exchange rates finds that when real exchange rates are modeled as a linear process, they exhibit nonstationary behavior. This has the implication that any shocks to real exchange rates are permanent which contradicts the concept of Purchasing Power Parity (PPP). Rogoff (1996) refers to this phenomenon as the 'PPP puzzle', since it casts doubt on the validity of a linear process for exchange rate movements. The PPP puzzle refers to two key findings that appear to be inconsistent: large short run volatility of real exchange rates which appear to be inconsistent with the long run stability observed in real exchange rates.

Recent papers over the past decade argue that this puzzle can be resolved by modeling the series as nonlinear stationary as opposed to a linear stationary process. The nonlinear nature of the series arises because of transactions costs and noise trading (Michael Nobay, and Peel (1997) and Killian and Taylor (2003)). Inside a certain band, real exchange rates follow a random walk. However, as exchange rates approach and move beyond a certain threshold, they become, as predicted by PPP, mean reverting -- hence the nonlinear nature of the time series process.

Threshold models have been most often used to capture this nonlinear behavior. In particular, the ESTAR model has been largely popular, because this framework has the ability to adjust its dynamic behavior depending on how much the real bilateral exchange rate deviates from the PPP norm. Negative and positive deviations are treated symmetrically with this type of model. Thus, if deviations from the PPP determined norm are small, then the ESTAR framework will model them as being persistent. However, if the deviations are far from the norm or threshold, then the ESTAR model will exhibit mean reverting dynamics.

However there are problems with this framework. One problem, as pointed out by Killian and Taylor (2003) is that forecasts based on ESTAR models do not outperform a random 
walk. Moreover, as pointed out by van Dijk et al (1999), large outliers may cause a series to be mistaken as an ESTAR process even though true DGP is linear.

This paper questions the power of the main test designed to detect an ESTAR process. We find that in estimating several real exchange rate series as ESTAR processes, the appropriateness of the ESTAR model depends on the country in question. There are other factors besides noise traders and transactions costs that can explain why, for some countries, the real bilateral exchange rate displays nonstationary behavior. We proceed to investigate whether there are other thresholds, aside from some PPP determined real exchange rate target that are relevant.

We investigate cases for nonlinear-threshold models of real exchange rates, where lags of the exchange rate itself may not be the appropriate threshold. More specifically, in the case of the real dollar-sterling exchange rate, we find that the real exchange rate series is better represented by an open-loop TAR process that uses the spread between the US and the UK overnight interest rate as the threshold variable. In the case real dollar-lire exchange rate, we show that the nonstationary behavior results from structural breaks. Prior to this break, this exchange rate was nonstationary and after the break became linear-stationary. Moreover we argue the two periods for this exchange rate occurred not because of frictions but because of Italian macroeconomic policies.

The remainder of the paper is organized as follows. Section 2 reviews the recent literature leading to the models that incorporate nonlinear adjustment mechanisms. Section 3 develops the framework under which we examine and compare the predictions of the two setups. In section 4, we outline the estimation methodology and report estimation results. Section 5 examines alternative explanations for nonlinear behavior apart from a threshold in the real exchange rate. Section 6 concludes. 


\section{A Brief Review of the Literature}

This section touches on some of the main highlights in the PPP literature pertaining to whether bilateral real exchange rates follow a random walk or not. An excellent survey of the literature and the ensuing debate on PPP can be found in Taylor and Taylor (2004).

Studies, beginning in the 1980s, focusing on unit roots and cointegration, brought into question the validity of PPP. Such tests, by being unable to reject the null of a unit-root in real exchange rates, implied that real exchange rates followed a random walk ${ }^{1}$. Proponents of PPP were alarmed by these results because most reasonable theoretical models assumed that deviations from PPP should be temporary at best. Though there may be rigidities in domestic nominal prices, long term monetary neutrality implies that any effects of money shocks on the real exchange rate adjusted for price differential should die out in the long run.

An alternative view began to form in the late 1990s. This view purported that models of exchange rate determination need to incorporate the presence of frictions such as transactions costs(see, e.g., Michael Nobay and Peel (1997) or noise traders (Killian and Taylor (2003)). The idea being that because of frictions, small, permanent deviations from PPP were possible. However, large deviations would be corrected where the size of the adjustment (degree of autoregressive decay) depends positively on the size of the deviation. Real exchange rates become mean reverting only when there is a substantial deviation from purchasing power parity. Incorporating these concepts involved deriving and estimating nonlinear time series models. A particular framework that exhibited this type of nonlinear behavior was the exponential smooth transition autoregressive (ESTAR) model, which is a variant of the smooth transition auto regressive (STAR) models. This type of model has been relatively successful in explaining some observed characteristics of real exchange rates. Examples of papers that incorporate the STAR and ESTAR specification include Michael, Nobay and Peel (1997), Taylor, Peel and Sarno (2001), and Lothian and Taylor (2004).

${ }^{1}$ For unit-root test pertaining to bilateral and effective real exchange rates see Roll (1979), Adler \& Lehman (1983), Darby (1983), Edison (1985), Meese \& Rogoff (1988), Enders (1988), Mark (1990). For cointegration tests pertaining to bilateral and effective real exchange rates see Enders (1988), Taylor (1988), Mark (1990), and Patel (1990). 
Under these types of models, the degree of autoregressive decay continuously varies depending on the deviation from PPP norm. To summarize, the further the real exchange rate is from the PPP norm, the less effect transaction inhibiting frictions will have on the ability of the exchange rate to adjust towards the norm. The closer the exchange rate is to the norm, the more likely adjustment will be inhibited by transactions costs, and therefore causing the series to exhibit nonstationary behavior.

Complementing the use of the STAR framework is the methodology used to test for STARtype nonlinearity. First, according to this methodology, tests for nonstationary are performed (see Lothian and Taylor (2004)). Second, assuming that the null of nonstationarity is not rejected (otherwise, it is not necessary to go any further), the next step is to use the procedure suggested by Teräsvirta (1994) to determine whether the time series is linear or conforms to a STAR process. We outline the analytical framework in the next section, followed by the methodology used to estimate a STAR type process.

\section{The Analytical Framework}

In this section, we outline the framework for the nonlinear adjustment mechanisms used to explain bilateral real exchange rate movements.

Assuming no transaction costs, the long run PPP condition may be written as:

$$
e_{t}+p_{t}^{*}-p_{t}=s+y_{t}
$$

where $e_{t}$ is the logarithm of the nominal exchange rate (domestic price of foreign currency), and $\mathrm{p}_{\mathrm{t}}$ and $\mathrm{p}_{\mathrm{t}}{ }^{*}$ denote the logarithm of the domestic and foreign price levels at time $\mathrm{t}$ respectively. $s$ is a constant reflecting differences in units of measurement whilst $y_{t}$ is a stationary error term representing deviations from PPP. Defining the real exchange rate in logarithmic form as:

$$
q_{t} \equiv e_{t}+p_{t}^{*}-p_{t}
$$

It is easy to see by plugging the definitional equation (2) into (1) that the real exchange rate can be viewed as a measure of the deviation from PPP. 


\section{The Benchmark (Linear) Case}

The benchmark case considered within this paper is one where deviations from PPP follow a linear process as shown in (3) below:

$$
y_{t}=\alpha_{0}+\sum_{j=1}^{p} \alpha_{j} y_{t-j}+\varepsilon_{t}
$$

where $\alpha_{\mathrm{j}}$ are the coefficients to be estimated, and $\varepsilon_{\mathrm{t}}$ is a random error. If the PPP condition is to hold in the long run, then deviations from PPP should be stationary so that the process that generates $\mathrm{q}_{\mathrm{t}}$ should settle down in the long run to some equilibrium value. However, if $\sum_{j=1}^{p} \alpha_{j} \geq 1$, then the underlying data generating process for the real exchange rate contains a unit root, and hence the real exchange rate (or equivalently, deviations from PPP) are nonstationary. Early studies of PPP were unable to reject a unit root null hypothesis for $\alpha_{1}$, e.g. Mark (1990).

\section{The ESTAR Case}

Beginning in the 1990s it was shown that PPP could hold once frictions such as transactions costs and noise traders were accounted for. A family of models that could incorporate these frictions is the STAR family. Of this family, studies typically incorporate an ESTAR specification because, in addition to being able to account for frictions, it also posits that adjustments to PPP deviations are the same for positive and negative deviations from equilibrium. Modeling the deviations in equation (1) to follow an ESTAR process results in the following specification:

$$
y_{t}=k+\sum_{j=1}^{p} \theta_{j} y_{t-j}+\left(k^{*}+\sum_{j=1}^{p} \theta_{j}^{*} y_{t-j}\right) F\left(\gamma, y_{t-d}\right)+u_{t}
$$

where the sequence of deviations from PPP, $\left\{\mathrm{y}_{\mathrm{t}}\right\}$, is a stationary, ergodic process, $\mathrm{u}_{\mathrm{t}} \sim \mathrm{iid}\left(0, \sigma^{2}\right), \gamma \in[0,+\infty)$ and $\mathrm{F}($.$) represents the transition function from one regime to$ another and it determines the degree of mean reversion. The transition function is itself dependent upon the parameter $\gamma$ which determines the speed of adjustment between the regimes. Our preferred choice for the transition function arises from an assumption about deviations from PPP. Since economic intuition suggests that both positive and negative 
deviations from PPP would have symmetric effects on mean reversion, we formulate the transition function as an exponential function:

$$
F\left(\gamma, y_{t-d}\right)=1-\exp \left[-\gamma\left(y_{t-d}-c_{0}\right)^{2}\right]
$$

This exponential transition function, has the following properties: $\mathrm{F}:(0, \infty) \rightarrow[0,1], \mathrm{F}[0]=0$ and $\operatorname{Lim}_{x \rightarrow \pm \infty} F[x]=1$. It is symmetric around zero. An alternative formulation for the transition function would be the logistic transition function which would result in an LSTAR model, where the transition function would be written as:

$$
F\left(\gamma, y_{t-d}\right)=\frac{1}{1+\exp \left[-\gamma\left(y_{t-d}-c_{0}\right)\right]}
$$

However, this formulation for the transition function would imply that real exchange rate deviations were asymmetric, which we believe to be implausible given the current context and the previous literature on real exchange rates. ${ }^{2}$

Using (5) as the transition function in (4) results in an ESTAR model whose interpretation is as follows. When $\mathrm{y}_{\mathrm{t}-\mathrm{d}}=\mathrm{c}^{*}, \mathrm{~F}()=$.0 , and the model in (4) becomes a linear AR(p) model:

$$
y_{t}=k+\sum \theta_{j} y_{t-j}+u_{t}
$$

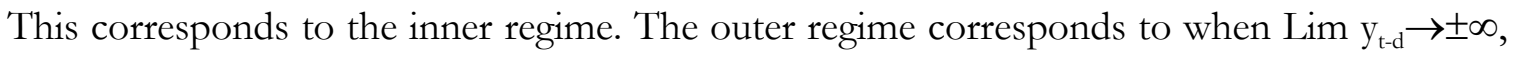
which yields $\mathrm{F}()=$.1 , and hence (5) becomes a different $\mathrm{AR}(\mathrm{p})$ model:

$$
y_{t}=\left(k+k^{*}\right)+\sum\left(\theta_{j}+\theta_{j}^{*}\right) y_{t-j}+u_{t}
$$

As long as $\theta^{*} \neq 0$ for some $\mathfrak{j}$, then this regime has a different speed of mean reversion. Another point of interest to note is that one of the features of the STAR family is that deviations from equilibrium yield increasing mean reversion but only after a delay, $d$. This can be seen by the inclusion of a d period delay in the deviations, $\mathrm{y}_{\mathrm{t}-\mathrm{d}}$ in equation (4).

Following Michael et al. (1997), we reparameterize the ESTAR model in (4) into an errorcorrection form:

\footnotetext{
${ }^{2}$ We wish to point out at this juncture that we leave choice of the transition function to use as the outcome of the STAR estimation methodology, rather than imposing the ESTAR transition function ourselves. More details follow regarding the choice in the next section.
} 


$$
\Delta y_{t}=k+\lambda y_{t-1}+\sum_{j=1}^{p} \phi_{j} \Delta y_{t-j}+\left(k^{*}+\lambda^{*} y_{t-1}+\sum_{j=1}^{p} \phi_{j}^{*} \Delta y_{t-j}\right) \times F\left(\gamma, y_{t-d}\right)+u_{t}
$$

The important parameters in this form are $\lambda$ and $\lambda^{*}$. It is easy to see from this specification that if there is any evidence of nonstationary behavior, either $\lambda$ or $\lambda^{*}$ will be greater than zero. However, if the outer regime is stationary and the inner regime nonstationary, then we must have $\lambda^{*}<0$ and $\lambda+\lambda^{*}<0$. In other words, if deviations from PPP are small, then $\mathrm{y}_{\mathrm{t}}$ may not be nonstationary. For large deviations from PPP, then the process for $y_{t}$ is mean reverting.

\section{Testing for Stationarity and Non Linearity}

In this section we analyze the real bilateral exchange rates between the United States and five of its trading partners - Canada, France, Italy, Japan, and the United Kingdom ${ }^{3}$. We first test each series for stationarity. If a series is found to be nonstationary, we then employ Teräsvirta's (1994) test to determine whether the series is linear. And if linearity is rejected we then employ the same test to determine what type of STAR process best describes the series.

\section{Data}

The data set consists of average monthly spot exchange rates observations for the US dollar against the UK sterling, French Franc, Canadian dollar, Italian Lira and the Japanese Yen as well as monthly observations on consumer price indices for the US, UK, France, Canada, Italy and Japan. The data taken from the International Monetary Fund's International Financial Statistics database covers the sample period 1973M01 to 2005M10 for the US, UK, Canada and Japan; for France and Italy, the sample period ends in 1998M12. Real exchange rates were constructed according to equation (4), where $e_{t}$ is taken as the logarithm of the dollar price of foreign currency (i.e. dollars per unit of foreign currency); $p_{t}$ is the logarithm of the US consumer price index, and $\mathrm{p}^{*}$ is the logarithm of the consumer price index in the other countries. We renormalize the real exchange rate (and the other series) so that in January

\footnotetext{
${ }^{3}$ According to the 2005 IMF Direction of Trade Statistics, Canada, Japan, and the U.K. make up three of the U.S.'s top four trading partners.
} 
1973, the log real exchange rate equals zero. Figure 1 depicts the spot dollar real exchange rate series against the countries mentioned above.

\section{Testing for Stationarity}

In estimating the linear model so that we may test for nonstationarity, we initially need to determine the order of autoregression, $\mathrm{p}$. This is done by utilizing an information criterion like the Akaike and Schwartz criteria as well as examining the partial autocorrelation function (PACF) for the real exchange rate. Table 1 shows the order of autoregression, $\mathrm{p}$, picked for the different real exchange rates. Having picked the order of autoregression, we then proceeded to estimate the linear AR(p) model given in equation (3):

$$
y_{t}=\alpha_{0}+\sum_{j=1}^{p} \alpha_{j} y_{t-j}+\varepsilon_{t}
$$

The results for the five bilateral real exchange rates we consider are shown in table 2. To test for stationarity, we employ the augmented Dickey-Fuller and Phillips Perron on equation (3) to determine which of any of the series are nonstationary. The results from these tests are outlined in Table 3.

As can be seen from the table, we are unable to reject the null hypothesis of a unit root in the real exchange rate series at a 5\% level of significance using both the augmented DickeyFuller and Phillips-Perron tests. In addition, the real exchange rate series appears to be difference-stationary. The finding here is consistent with the well known result that real bilateral exchange rates appear to exhibit nonstationary behavior. It should be noted however, that the failure to reject the null hypothesis of a unit root may be accounted for by the low power of unit root tests. Alternatively, it could also indicate the presence of nonlinear dynamics in the real exchange rate, a matter we will now investigate.

\section{ESTAR Estimation}

To specify a STAR model, it is necessary to first determine the order of the autoregression parameter, $\mathrm{p}$. We utilize the same values for $\mathrm{p}$ as we did in the linear case. Once the order, $\mathrm{p}$, is determined there are three further steps: testing for linearity; selecting the value for the delay parameter, $d$; and choosing between an LSTAR and an ESTAR specification. These further three steps involve employing a method suggested by Teräsvirta (1994). The idea behind these tests is to take a third order Taylor expansion of the transition function given in 
equation (4) around $\gamma=0$. Given $\mathrm{p}$, this boils down to estimating the auxiliary regression below for a fixed parameter $\mathrm{d}$ :

$$
y_{t}=\beta_{00}+\sum_{j=1}^{p}\left(\beta_{0 j} y_{t-j}+\beta_{1 j} y_{t-j} y_{t-d}+\beta_{2 j} y_{t-j} y_{t-d}^{2}+\beta_{3 j} y_{t-j} y_{t-d}^{3}\right)+\varepsilon_{t}
$$

The linearity test involves testing the joint hypothesis that all the coefficients correspond to the cross products in (7) are zero:

$$
\mathrm{H}_{0, \mathrm{LIN}}: \beta_{1 j}=\beta_{2 j}=\beta_{3 j}=0 \quad(\mathrm{j}=1, \ldots, \mathrm{p})
$$

The above test is also used to determine the delay length, $\mathrm{d}$. This involves running the linearity tests for all plausible values of $d$ and picking the value of $d$ that minimizes the test's p-value (as suggested by Tsay, 1989). Because we utilize monthly data, we consider values of $\mathrm{d}=1, \ldots, 12$ as plausible values for the delay parameter. The results from the linearity tests are reported in Table (4).

The main results from table 4 are as follows. From panel A of the table, it can be seen that we are unable to reject the null of linearity for US-Canada and US-Japan. For the others, we reject linearity and select $\mathrm{d}=11$ for US-France, $\mathrm{d}=10$ for US-Italy and $\mathrm{d}=7$ for US-UK. For the three nonlinear series, the next step is to determine whether to specify them as LSTAR or ESTAR models. To do so, following Terasvirta's recommendations, we test the following hypothesis corresponding to equation (7):

$$
\begin{array}{lll}
\mathrm{H}_{0,1}: \beta_{3 j}=0 & & (\mathrm{j}=1, \ldots, \mathrm{p}) \\
\mathrm{H}_{0,2}: \beta_{2 j}=0 & \mid \beta_{3 j}=0 & (\mathrm{j}=1, \ldots, \mathrm{p}) \\
\mathrm{H}_{0,3}: \beta_{1 j}=0 & \mid \beta_{2 j}=\beta_{3 j}=0 & (\mathrm{j}=1, \ldots, \mathrm{p})
\end{array}
$$

If the p-value resulting from the hypothesis test in equation (8c) is the smallest, we pick the ESTAR specification otherwise the decision rule suggests an LSTAR specification. ${ }^{4}$ According to the results reported in Table 4b, all three of the nonlinear processes are ESTAR models.

\footnotetext{
${ }^{4}$ Details for the reasoning behind the decision rule are outlined in Teräsvirta (1994).
} 
The next step is to estimate the ESTAR model for each of the bilateral real exchange rate series using nonlinear least squares. This process yields estimators which are consistent and asymptotically normal. The results are reported in Table $5 .{ }^{5}$ For US-France, we find that the estimated value of $\lambda$ is positive, but not significant at the 10 percent level, although it is fairly close. This indicates that we cannot reject the null of nonstationary behavior within the inner regime. However, $\lambda^{*}$ is both negative and greater in magnitude than $\lambda$ and according to our estimated results, the hypothesis that $\lambda+\lambda^{*} \geq 0$ is rejected at a 0.008 level of significance. Hence, we find evidence of stationary behavior in the outer regime and the dollar-franc real exchange rate appears to be nonlinear and globally stationary. We find similar results for Italy where the hypothesis that $\lambda+\lambda^{*} \geq 0$ is rejected at a 0.08 level of significance. However for the US-UK, we find that we are unable to reject the null hypothesis that either $\lambda=\lambda^{*}=$ 0 . Moreover, when looking at the estimated AR parameters for the outer regime, i.e. $\phi_{j}^{*}$ $(j=1, \ldots, p)$, we find that none of them appear to be significant for any of the countries with the exception of Italy. The only significant parameters appear in the inner regime.

In addition, the results for Italy appear to be sensitive to the initial vector of parameters used to initiate the nonlinear least squares search. Changing the starting value for the threshold parameter, $\mathrm{c}_{0}$, yields a different ESTAR specification. This specification had a lower sum of squared residuals of 0.169 , but yields parameter estimates that appear to be counter-intuitive: $\lambda$ is significantly less than zero and $\lambda^{*}$ is significantly greater than zero. This would indicate that the further the real dollar-lira exchange rate is from its threshold, the more nonstationary it is, whilst the closer the real exchange rate is to its threshold, the greater the degree of stationarity. We present the preferred specification in Table 5, but point out that there is nothing within the estimation methodology that allows us to discard the alternative specification that was estimated, apart from the idea that it runs contrary to the way we would expect real exchange rates to behave under this framework.

These results highlight and motivate the key insight provided by this paper. The STAR estimation methodology that is advocated by the literature and by which we follow, suggests

\footnotetext{
${ }^{5}$ In estimating the model through nonlinear least squares, we follow a suggestion by Teräsvirta (1994) and normalize the exponent of the transition function by the sample variance of $y$. This then provides $\gamma=1$ as an appropriate starting value for the gamma parameter in the nonlinear least squares procedure.
} 
the US-UK, US-France and US-Italy data are nonlinear and follow an ESTAR process. However, in actually estimating the model, the parameter estimates suggest that the ESTAR model is not a valid process for dollar-sterling real exchange rate, whilst the estimates for the dollar-lira real exchange rate appear to hinge on the initial conditions for the threshold parameter. It is only in the US-France case that we are able to estimate an ESTAR specification, yet several of the parameters in the outer regime are found to be insignificant. It appears that we are only able to identify and estimate parameters in the inner regime without any issue.

One possibility is that the ESTAR model and the diagnostic tests suggested by Teräsvirta (1994) are falsely rejecting the linearity hypothesis. According to van Dijk et al (1999) the presence of outliers causes Teräsvirta's test to falsely reject the null hypothesis. To investigate this possibility we analyze the standardized residuals of the linear model whose results are reported earlier in this section. The results are presented in figure 2 , where we also present plots for the other countries as a matter of interest. It is interesting to note that there are only a small number of observations where the standardized residuals lie outside three standard deviations across all countries. For both the US-UK and US-Italy, there were three standardized residuals that have a magnitude exceeding 3.0. In the case of the US-UK, there occurred in 1979:07 (-3.66), 1985:04 (4.07), and 1992:10 (-3.84). For US-Italy, there occurred in 1982:06 and in 1991:03, and 1992:10.

If we re-estimate the linear model after dummying out these three observations, we obtain the results that are reported in table 6. For the US-UK case, the major change can be seen in terms of the sum of squared residuals. The sum of squared residuals in the original linear model was 0.227. After dummying out the observations pertaining to 1979:07, 1985:04 and 1992:10, the sum of squared residuals falls to 0.200 - a more than 9.7 percent decline. Just dummying out the largest outlier (1985:04) reduces the sum of squared residuals by 4.5 percent to 0.2167. The sum of squared residuals for the ESTAR model was 0.212. Just dummying out the largest observation reduces the sum of squared residuals by more than modeling the exchange rate as an ESTAR process. 
In the case of US-Italy, the sum of squared residuals in the original linear model was 0.181 . After dummying out the three observations pertaining to 1982:06, 1991:03, and 1992:10, the sum of squared residuals falls by 9.4 percent from 0.181 to 0.164 which is slightly less than the sum of squared residuals obtained from the US-France ESTAR model (0.169).

At issue is whether the presence of outliers, or influential observations in the data cause the diagnostic tests suggested by Teräsvirta (1994) to falsely reject the linearity hypothesis in favor of an ESTAR model and hence, is the estimated STAR effect simply a consequence of just a few outliers. The methodology behind estimating ESTAR (and in general, TAR) models requires sufficient observations in different regimes to be able to correctly estimate the underlying parameters in those regimes without introducing biases or efficiency losses in estimating them. If we assume that the influential observations denoted in the figures above represent valid observations in the "outer" regimes of a true STAR process, then the results here would seem to indicate that we only have a few observations which we can use to make inferences about parameters in that regime. This is an issue since the lack of observations would imply that any estimation procedure would be subject to small sample biases. This idea is pursued by Ahmad (2007).

If we hold the above to be true, then in some sense, the ESTAR modeling methodology requires a certain number of "outliers" to be able to correctly identify and estimate the underlying model. If there are insufficient deviations from the threshold value(s), then we have an identification issue, since we will have insufficient observations in the outer regimes and hence we will be unable to correctly identify the different regimes. In the case of the two real exchange rates discussed above, we find that the lack of enough observations in the outer-regimes provides a possible explanation as to why the linear models for these exchange rates perhaps captures movements in the real exchange rate better than the nonlinear (ESTAR) model. It also provides an alternative explanation for why we were unable to reject the null hypothesis of linearity for Canada and Japan. Indeed, of the five real exchange rate series we examine, only the dollar-franc series is able to be estimated as an ESTAR process without any problems (although the parameters in the outer regime appear to be insignificant). 


\section{Some Simulation Results}

An extension to this idea is to consider whether data possess enough observations to be able to correctly infer different regimes, given that the underlying data generating process contains nonlinear dynamics. Are we able to correctly infer the true form of the nonlinear dynamics? Similar results were found by Ahmad (2007) who examines this issue in the context of small sample bias for Threshold autoregressive models. To highlight this idea further, we proceed to investigate further through a Monte Carlo investigation.

We start by using our estimates of the coefficient arising out of the linear AR(p) model for the dollar-sterling real exchange rate. Utilizing these coefficients, we then generate pseudo data that has the same characteristics of the actual real exchange rate, (i.e. the same mean, variance, and sample size), but where the underlying data generating process is linear. For each trial, we discard the first 200 observations and then implement the ESTAR methodology on the remaining observations to see if: (i) linearity is accepted or rejected; (ii) whether the methodology we implemented here in this paper (falsely) indicates an ESTAR or LSTAR specification, given that it has rejected linearity. We do this for 100, 500, and 1,000 trials where we set the size of the test at a 5\% level of significance. The results are reported in the table 7 .

As can be seen from the table, there are substantial distortions in the size of the test. That is, given a true null hypothesis that the data generating process is linear, we find that we reject the true linear null and favor the alternative of nonlinearity at a rate substantially higher than that of 5 percent. The results here give credence to the point made by van Dijk et al (1999) that influential observations in the data may distort the power of the tests used to detect the presence of nonlinearities in the data.

\section{Do exchange rates follow a nonlinear process?}

We have suggested that Teräsvirta's (1994) test suggests an ESTAR process in three out of five cases. However our findings above call into question the appropriateness of the ESTAR model in two of the three cases, i.e. the dollar-sterling and the dollar-lira real exchange rates may not be ESTAR type processes. If that is the case and the reader is at least partially 
convinced, then a natural question arises. If they are not ESTAR processes, what are they? The next step is to then determine whether this is because these processes are truly linear, or whether they are nonlinear albeit not STAR type processes. Certainly the simulation results above would indicate that it might not be uncommon for the test size to be distorted, which in turn could cause a linear process to be mistaken for a nonlinear STAR process.

We would expect that once these influential observations are removed, the series would conform to a linear model if the problem arises due to influential observations. Hence, we proceed by removing the influential observations from both the dollar-sterling and the dollar-lira series and reapply Teräsvirta's test. The results are shown in table 8. In both cases the null hypothesis of linearity is rejected. In addition, the test results suggest an LSTAR model for both series based on Teräsvirta's model selection criterion. However, when we estimate both series as an LSTAR model, we find that we are unable to reject the hypothesis that $\lambda+\lambda^{*} \geq 0$ for either series. ${ }^{6}$ From this, we can form one of two conclusions. First, we could conclude that influential observations may not be the only reason for rejecting linearity in favor of nonlinearity. Alternatively, it may be that the underlying data generating process is inherently nonlinear, and that these influential observations are drawn from the set of observations that truly lie in the outer regime. However, it may be the case that the total number of observation in that regime is limited, which would mean that we are unable to capture the true nature of the nonlinear dynamics correctly.

Given the results above, it is worth exploring whether these series adhere to an alternative nonlinear specification. More specifically we look at Threshold Autoregressive (TAR) models. Like STAR models, the dynamics of the process depends on where the exchange rate is relative to a threshold variable. However, instead of assuming a continuous adjustment, TAR models assume that the adjustment is discrete. In addition, instead of specifying the threshold variable to be the value of the real exchange rate lagged $\mathrm{d}$ periods, we look to economic theory in search for where the threshold may lie.

Several models of exchange rate determination, e.g. the monetary model of exchange rate determination amongst others, suggest that exchange rates are determined as a result of

\footnotetext{
${ }^{6}$ Estimation results are not reported here.
} 
monetary policy actions by central banks, particularly within a floating exchange rate system. Hence, we consider the case where the underlying threshold variable does not lie within the exchange rate domain, but is instead dependent upon a monetary variable that influences the real exchange rate. In the case of the dollar-sterling real exchange rate, the threshold variable is found to be within the spread between the federal funds rate and the overnight interest rate in the UK. In the case of dollar-lira real exchange rate, we find a similar threshold within the spread between the log of the U.S. CPI and the Italian CPI.

We use a method suggested by Tsay (1989) to test for threshold nonlinearity. This entails rearranging the autoregression according to the order of a threshold variable - the spread between the overnight interest rates in the U.S. and U.K. in the case of the real dollarsterling exchange rate, and the spread between the natural logs of the U.S. and Italian consumer price indices in the case of the real dollar-lira rate. Tsay's (1989) test then involves searching for a break in this ordered autoregression, which would indicate the presence of a threshold.

\section{The Dollar-Sterling TAR Model}

The threshold variable we use for the dollar-sterling exchange rate is the absolute value of the previous month's spread between the U.S. federal funds rate and the U.K. overnight rate. Using Tsay's arranged autoregression test, we obtain a test statistic of $\mathrm{F}(4,382)=4.8$ with a corresponding $\mathrm{p}$-value of 0.00087 . The result suggests that the null hypothesis of linearity can be rejected for any reasonable level of significance. According to this method, the threshold level for the spread is 4.66 percentage points.

It is interesting to note, that the spread between the UK and US overnight rates was greater than 4.66 percentage points in each of the three periods that we documented as outliers earlier. In 1979:07, 1985:04 and 1992:10, the lagged difference between the US and UK overnight rates were 5.29, 5.67 and 6.03 percentage points respectively. Thus, we would argue that these outliers are indicative of a nonlinear process albeit not an ESTAR process.

Based on this information, we estimate the following threshold autoregression model: 


$$
\begin{aligned}
& y_{t}=D\left(\alpha_{0}+\sum_{j=1}^{2} \alpha_{j} y_{t-j}+(1-D)\left(\alpha_{0}+\sum_{j=1}^{3} \alpha_{j} y_{t-j}\right)+\varepsilon_{t}\right. \\
& D_{t}=\left\{\begin{array}{l}
1 \text { if } x_{t} \geq 4.66 \% \\
0 \text { if } x_{t}<4.66 \%
\end{array}\right.
\end{aligned}
$$

where $\mathrm{x}_{\mathrm{t}}$ represents the interest rate differential between the overnight interest rate in the US and that in the UK. These results, reported in table 9, indicate that the real dollar-sterling exchange rate is more persistent when the lagged spread between US and UK over-night rates are below the threshold of $4.66 \%$ than when they are above it. In addition, 83 out of the 393 observations occurred when the spread between the two overnight rates were above 4.66 percent. It is also interesting to note that the sum of squared residuals, 0.2090 in this model is less than the sum of square residuals in both the linear and the ESTAR models.

\section{The Dollar-Lira TAR Model}

For the dollar-lira exchange rate, we find evidence of a threshold in the spread between the $\log$ of the U.S. CPI and the Italian CPI. Using Tsay's arranged autoregression test, we obtain a test statistic of $F(5,298)=2.7$ with a corresponding p-value of 0.021 . According to this method, the threshold level for the spread between the logs of the Italian and the U. S. CPI is 100.6 with four month delay. This threshold occurs at the point when Italy left the EMS in September of 1992. Italy remained above this threshold until the conversion to the Euro. We find similar results by conducting a QLR test for structural breaks with an unknown breakpoint. ${ }^{7}$ These results suggest that the relative price structure between Italy and the U.S. stabilized when Italy left the ERM. Perhaps the discipline from being part of the ERM, as well as Italy's commitment to rejoin the ERM after leaving it, enabled the Italian central bank to credibly conduct policy so that price stability was maintained and consequently fluctuations to the dollar-lire exchange rate were minimized.

We proceed to estimate the threshold autoregression model as:

\footnotetext{
${ }^{7}$ The latter test yields a significant F-statistic of F $=8.30$ in August of 1992 .
} 


$$
\begin{aligned}
& y_{t}=D \sum_{j=1}^{3} \alpha_{j} y_{t-j}+(1-D)\left(\sum_{j=1}^{2} \alpha_{j} y_{t-j}\right)+\varepsilon_{t} \\
& D_{t}=\left\{\begin{array}{l}
1 \text { if } x_{t} \geq 100.611 \\
0 \text { if } x_{t}<100.611
\end{array}\right.
\end{aligned}
$$

where $x_{t}$ represents the difference between the logs of the US CPI and the Italian CPI. The results from the estimation, which are also presented in Table 9, show that the dollar-lira real exchange rate is less persistent four months after the spread between the logs of U.S. and Italian CPI is crossed the threshold level of 100.7 which began in September of 1992. The SSR for this model is lower than both the linear and the ESTAR model for the real dollar lire exchange rate.

\section{Conclusion}

The recent literature on real exchange rates has adopted nonlinear methods to capture the dynamics of real exchange rates. These models are able to generate a rich array of dynamics, since the dynamic behavior depends upon the extent to which the current value of the real exchange rate differs from certain threshold points. The existence of these thresholds, which are motivated for example by transaction costs and noise traders, lead real exchange rates to exhibit nonstationary behavior within certain regimes, whilst they exhibit stationary behavior in other regimes. One of the more common approaches has been to utilize the smooth transition autoregressive (STAR) framework which links the persistence of the real exchange rate to the extent to which the real exchange rate has deviated from a PPP defined norm.

Within this paper, we investigate the underlying methodology used to detect STAR type behavior and try to determine whether the STAR framework appropriately captures the nature of the nonlinear dynamics exhibited by five spot dollar real exchange rates. The underlying methodology that is used to detect nonlinearity and STAR type behavior, suggests that three of the five real exchange rate series may be modeled as an exponential STAR (ESTAR) process. However, we find that a linear model that accounts for influential observations manages to fit the data better for two of these three real exchange rate series - 
the dollar-sterling and the dollar-lira series - and that overall, of the five real exchange rate series we investigate, four of the five appear to be linear.

We attribute these findings to the presence of the aforementioned influential observations, which we believe leads to distortions in the test statistics used to diagnose and detect the presence of nonlinearities in the data. This is supported by simulations we conduct that show significant distortions in the size of test statistics. Since our view is that the underlying series are nonlinear, we investigate the dollar-sterling and dollar-lira real exchange rates further and find that they are best modeled as open-loop TAR processes. The underlying threshold does not appear to be within the exchange rate domain, but rather within variables that pertain to monetary policy: in the overnight interest rate spread for US-UK and in the CPI spread for US-Italy.

The results of this paper are intended to provide a note of caution to the growing literature that utilizes STAR type frameworks to investigate real exchange rate behavior. We question whether this framework adequately captures the nonlinear nature of real exchange rates. We continue to hold to the view that real exchange rates exhibit nonlinear dynamics. However, based on our findings, we suggest that we may need to need to look at macroeconomic theory to determine where threshold points may lie, rather than assuming that they lie within the domain of lagged real exchange rates. 


\section{References}

Adler, M. and Lehman, B. (1983) "Deviations From PPP In The Long Run", Journal of Finance, Vol. 38, No. 4, December 1983, pp. 1471-87.

Ahmad, Yamin (2007) "Effects of Small Sample Bias in Threshold Models", mimeo

Darby, M. R. (1983) "Movements In PPP: The Short And Long Runs", in Darby and Lothian (eds.), The International Transmission Of Inflation, Chicago: University of Chicago Press.

Edison, H. J. (1985) “PPP: A Quantitative Reassessment Of The 1920s Experience”, Journal Of International Money And Finance, Vol. 4, September 1985, pp. 361-72.

Enders, W. (1988) “ARIMA and Cointegration Tests Of PPP Under Fixed And Flexible Exchange

Rate Regimes", Review of Economics and Statistics, Vol. 70, August 1988, pp. 504-08.

Kilian, L. and M. P. Taylor (2003) "Why is it So Difficult to Beat the Random Walk Forecast of

Exchange Rates?”, Journal of International Economics, Vol. 60, No. 1, pp. 85-107.

Lothian, J. R. and Taylor, M. P. (2004), "Real Exchange Rates Over The Past Two Centuries: How Important is the Harrod-Balassa-Samuelson Effect?”, Working Paper, 2004.

Mark, N. C. (1990) "Real And Nominal Exchange Rates In The Long Run: An Empirical Investigation", Journal Of International Economics, Vol. 28, February 1990, pp. 115-36.

Meese, R. A. and Rogoff, K. (1988) "Was It Real? The Exchange Rate Interest Differential Relation Over The Modern Floating Period", Journal Of Finance, Vol.43, No. 4, September 1988, pp. 933-48.

Michael, P., Nobay, R. A. and Peel, D. A. (1997) “Transaction Costs And Nonlinear Adjustments In Real Exchange Rates: An Empirical Investigation”, Journal Of Political Economy, Vol. 105, No. 4, August 1997, pp. 862-79.

Patel, J. (1990) “Purchasing Power Parity As A Long Run Relation", Journal Of Applied Econometrics, Vol. 57, October- December 1990, pp. 367-79.

Rogoff, K. (1996) “The Purchasing Power Parity Puzzle”, Journal Of Economic Literature, Vol. 34, June 1996, pp. 647-68.

Roll, R. (1979) "Violations Of PPP And Their Implications For Efficient Internationally Commodity Markets" in Marshall Sarnat and Giorgio P. Szego (eds.), International Finance And Trade Vol. 1, Cambridge, Massachusetts: Ballinger, pp. 133-176.

Taylor, A. M. and M. P. Taylor (2004) “The Purchasing Power Parity Debate”, Journal of Economic Perspectives, Vol. 18, No. 4, pp. 135-158.

Taylor, M. P. (1988) “An Empirical Examination Of Long Run PPP Using Cointegration Techniques", Applied Economics, Vol. 20, October 1988, pp. 1369-81. 
-----------, D. Peel and L. Sarno (2001) "Nonlinear mean reversion in Real Exchange Rates:

Towards a Solution to the Purchasing Power Parity Puzzles." International Economic Review, Vol. 42, No. 4, pp. 1015-1042.

Teräsvirta, T. (1994) "Specification, Estimation, and Evaluation of Smooth Transition Autoregressive Models", Journal of American Statistics Association, Vol. 89, pp. 208-218.

Tsay, R. (1989) “Testing and Modeling Threshold Autoregressive Processes.”, Journal of American Statistics Association, Vol. 84, (March 1989), pp. 231-240.

van Dijk, D.; Franses, P.H.; Lucas, A. (1999) “Testing for smooth transition nonlinearity in the presence of additive outliers," Journal of Business and Economic Statistics 1999, 17, 217-235. 


\section{Figures}
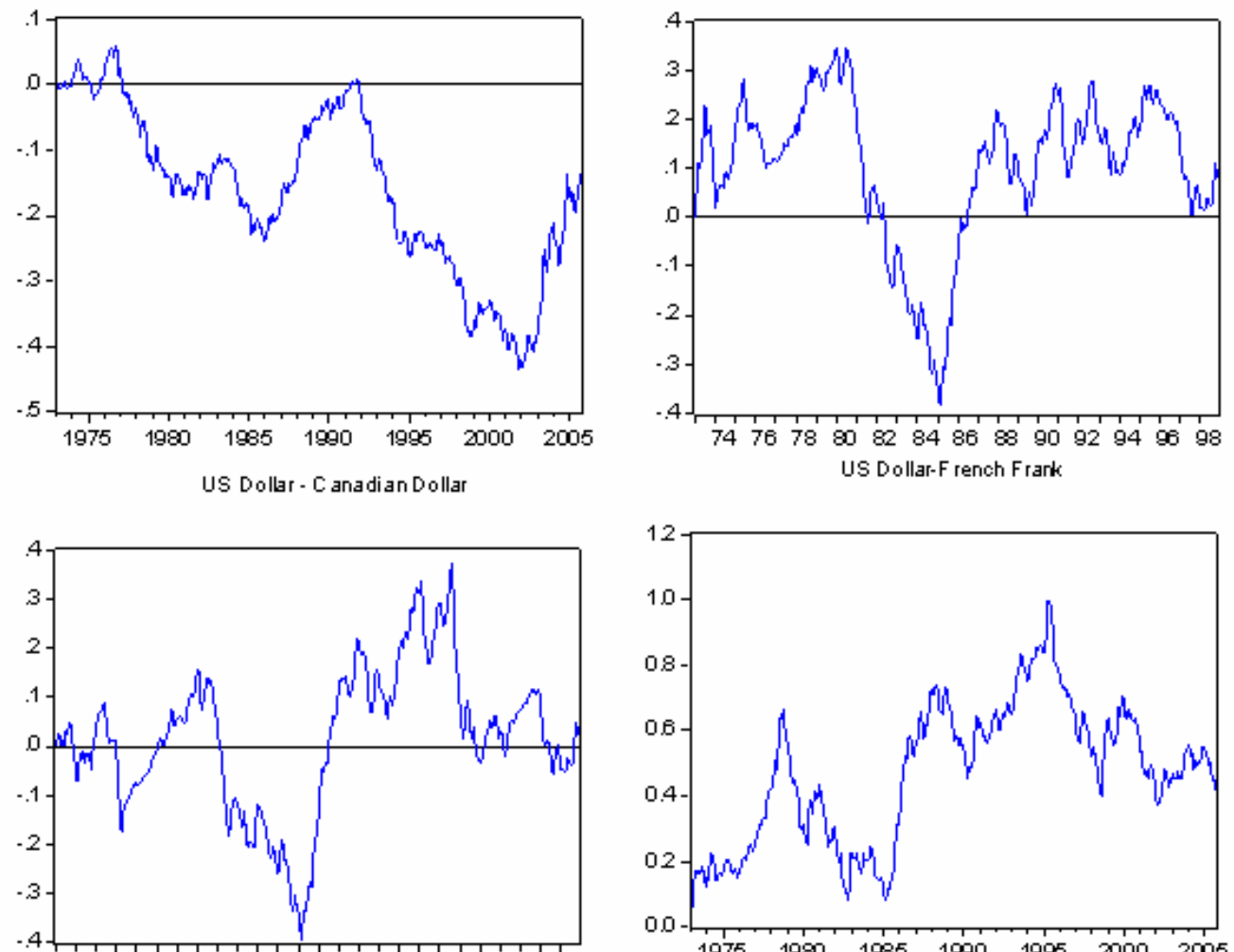

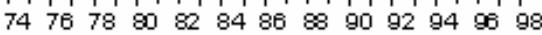
US Dollar - Halian Lir a
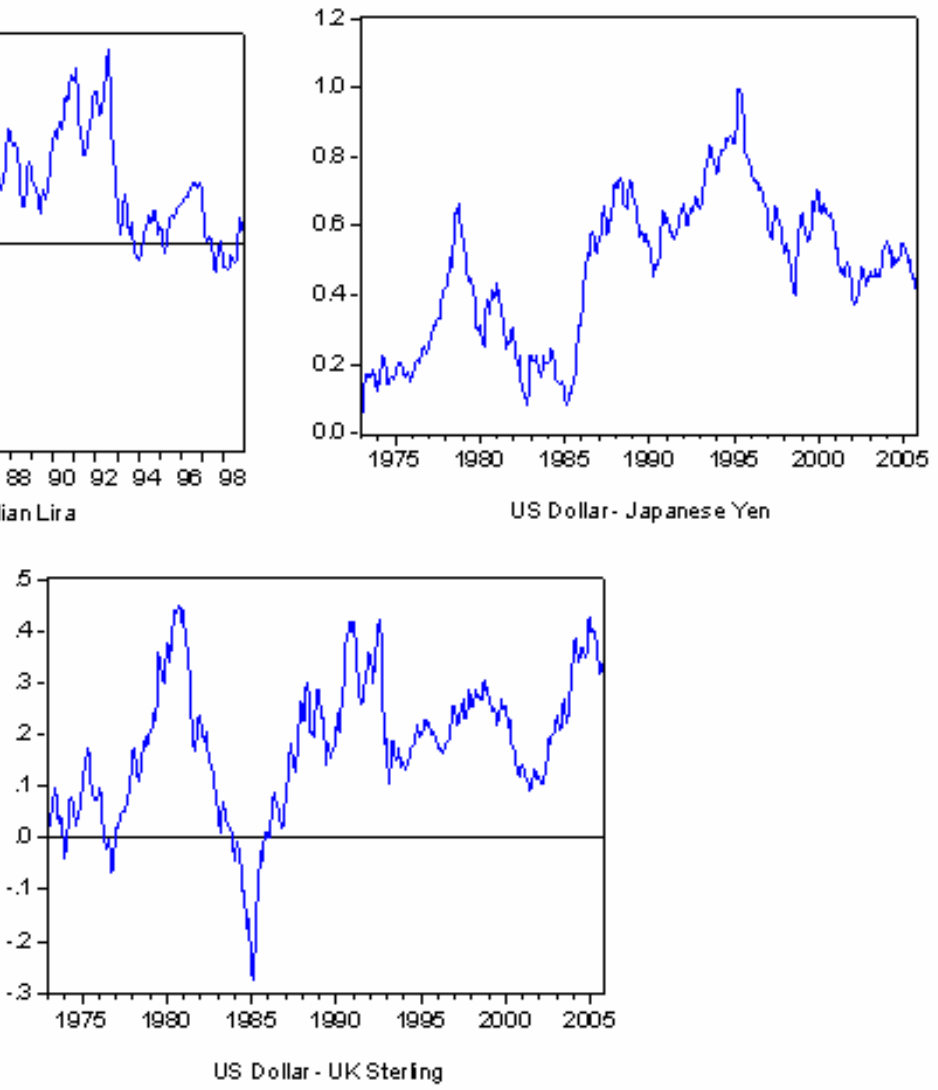

Figure 1: The spot dollax real exchange rates (rebased to 1973:1) 

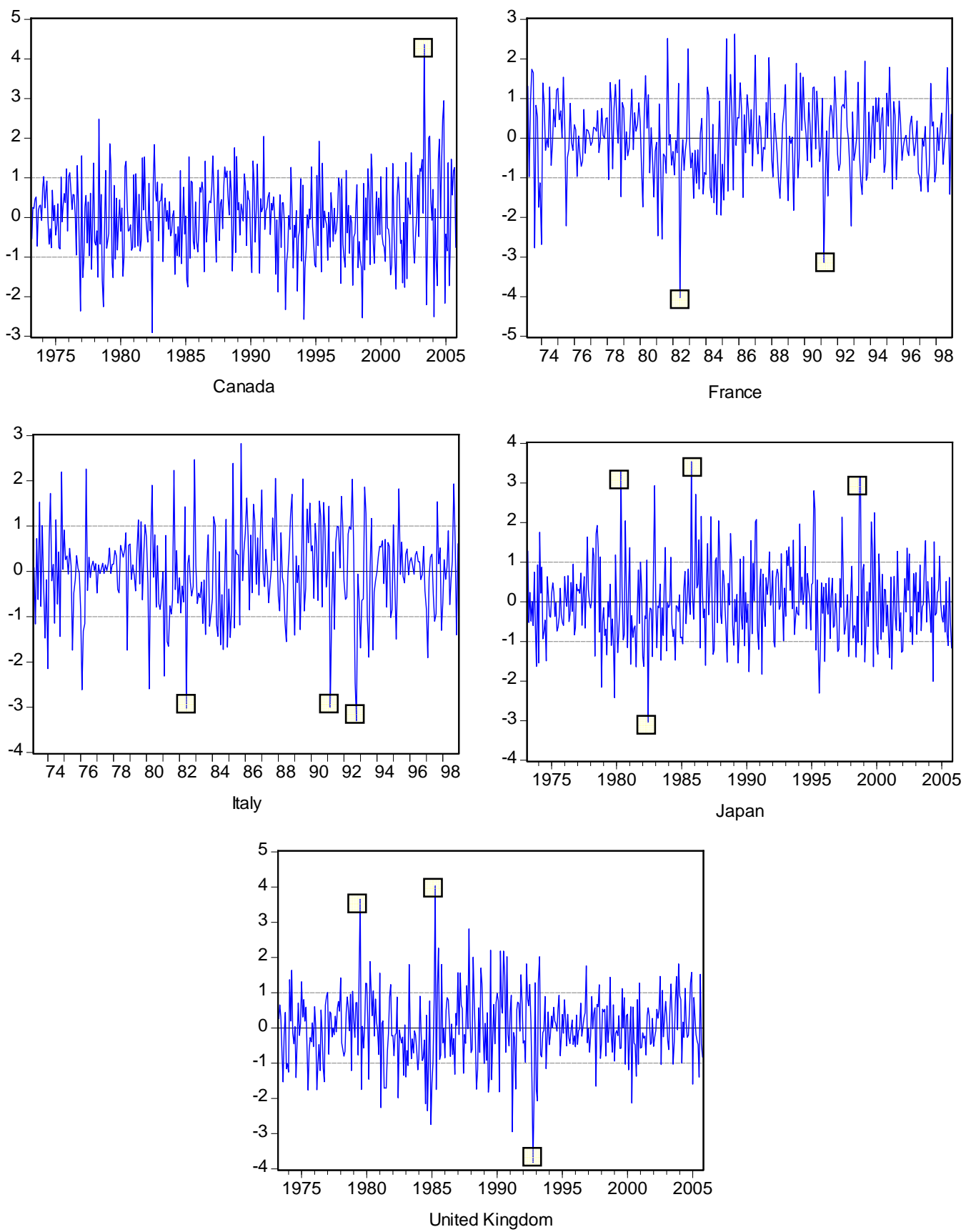

Figure 2: A plot of the standardized residuals with significant outliers 


\section{Tables}

\begin{tabular}{|c|c|c|c|c|c|c|c|}
\hline & \multirow{2}{*}{$\begin{array}{l}\text { AR }(p) \text { Lag } \\
\text { Selected } \\
\end{array}$} & \multicolumn{2}{|c|}{$\begin{array}{l}\text { Lag Length Suggested by } \\
\text { Information Criterion }\end{array}$} & \multicolumn{2}{|c|}{$\begin{array}{c}\text { Value of Information Criterion } \\
\text { at selected lag }\end{array}$} & \multirow{2}{*}{$\begin{array}{c}\text { Ljung - Box } \\
\text { Q-statistic }\end{array}$} & \multirow[b]{2}{*}{$\mathrm{p}$-value } \\
\hline & & AIC & SBC & AIC & SBC & & \\
\hline Canada & 2 & 11 & 2 & -6.004 & -5.973 & 0.170 & 0.919 \\
\hline France & 4 & 8 & 2 & -4.489 & -4.428 & 1.140 & 0.888 \\
\hline Italy & 2 & 2 & 2 & -4.586 & -4.550 & 0.546 & 0.761 \\
\hline Japan & 2 & 2 & 2 & -4.324 & -4.294 & 1.987 & 0.370 \\
\hline UK & 3 & 4 & 2 & 4.615 & 4.656 & 3.097 & 0.377 \\
\hline
\end{tabular}

Table 1: Lag length selection based on PACF, AIC and SBC criteria.

\begin{tabular}{lccccc} 
Variable & US-CAN & US-FRA & US-ITA & US-JAP & US-UK \\
\hline $\mathrm{p}$ & 2 & 4 & 2 & 2 & 3 \\
$\phi_{0}$ & -0.002 & 0.002 & 0.000 & 0.007 & 0.004 \\
& $(-1.532)$ & $(1.267)$ & $(0.244)$ & $(2.175)$ & $(2.169)$ \\
$\mathrm{y}_{\mathrm{t}-1}$ & 1.193 & 1.283 & 1.321 & 1.283 & 1.343 \\
& $(24.011)$ & $(22.623)$ & $(24.645)$ & $(26.698)$ & $(26.579)$ \\
$\mathrm{y}_{\mathrm{t}-2}$ & -0.200 & -0.401 & -0.342 & -0.298 & -0.484 \\
& $(-4.041)$ & $(-4.368)$ & $(-6.376)$ & $(-6.236)$ & $(-5.946)$ \\
$\mathrm{y}_{\mathrm{t}-3}$ & & 0.250 & & & 0.118 \\
& & $(2.710)$ & & & $(2.345)$ \\
$\mathrm{y}_{\mathrm{t}-4}$ & & -0.154 & & & \\
& & $(-2.729)$ & & & \\
\hline \hline & & & & & \\
$\mathrm{N}$ & & 312 & 312 & 394 & 394 \\
$\mathrm{R}^{2}$ & 394 & 0.972 & 0.973 & 0.983 & 0.967 \\
Sum squared resid & 0.991 & 0.196 & 0.181 & 0.299 & 0.227 \\
Standard error & 0.0120 & 0.0254 & 0.0243 & 0.0277 & 0.0242 \\
$\mathrm{Q}(p)$ & 0.170 & 1.140 & 0.546 & 1.987 & 3.097 \\
Durbin Watson & 1.989 & 1.989 & 1.970 & 1.976 & 1.977 \\
\hline \hline
\end{tabular}

Note:- The numbers in parenthesis are t-ratios. $Q(p)$ is the Ljung-Box (1979) statistic that is replicated here from table (2) and is distributed as a $\chi_{(\mathrm{p})}^{2}$ statistic. The critical values for the $\chi_{(2)}^{2}$ is 5.99 ; for $\mathrm{a} \chi_{(3)}^{2}$ is 7.81 , for a $\chi_{(4)}^{2}$ is 9.49

Table 2: Linear Autoregressive Model: Equation (3) 
Test Statistics (P-Value)

\begin{tabular}{|c|c|c|c|c|c|}
\hline & & $q^{A}$ & $\Delta q^{A}$ & $\mathrm{q}^{\mathrm{pp}}$ & $\Delta \mathrm{q}^{\mathrm{pp}}$ \\
\hline Canada & & $\begin{array}{l}-1.549 \\
(0.508)\end{array}$ & $\begin{array}{c}-16.151 \\
(0.000)\end{array}$ & $\begin{array}{c}-1.505 \\
0.530\end{array}$ & $\begin{array}{c}-16.159 \\
0.000\end{array}$ \\
\hline France & & $\begin{array}{l}-2.094 \\
(0.247)\end{array}$ & $\begin{array}{r}-13.364 \\
(0.000)\end{array}$ & $\begin{array}{l}-2.130 \\
(0.233)\end{array}$ & $\begin{array}{l}-13.338 \\
(0.000)\end{array}$ \\
\hline Italy & & $\begin{array}{l}-2.209 \\
(0.204)\end{array}$ & $\begin{array}{r}-12.449 \\
(0.000)\end{array}$ & $\begin{array}{l}-2.041 \\
(0.269)\end{array}$ & $\begin{array}{l}-12.449 \\
(0.000)\end{array}$ \\
\hline Japan & & $\begin{array}{c}-2.222 \\
(0.199)\end{array}$ & $\begin{array}{c}-14.684 \\
(0.000)\end{array}$ & $\begin{array}{l}-2.422 \\
(0.136)\end{array}$ & $\begin{array}{r}-14.610 \\
(0.000)\end{array}$ \\
\hline United Kingdom & & $\begin{array}{l}-2.723 \\
(0.071)\end{array}$ & $\begin{array}{c}-13.181 \\
(0.000)\end{array}$ & $\begin{array}{l}-2.464 \\
(0.125)\end{array}$ & $\begin{array}{r}-13.973 \\
(0.000)\end{array}$ \\
\hline Critical Values & $\begin{array}{l}1 \% \\
5 \% \\
10 \%\end{array}$ & $\begin{array}{l}-3.447 \\
-2.869 \\
-2.571\end{array}$ & & & \\
\hline
\end{tabular}

Notes: Statistics are Augmented Dickey-Fuller (ADF) and Phillips-Perron (PP) test statistics for the null hypothesis of a unit root process; the superscript (A) refers to the ADF whilst the (PP) superscript refers to the Phillips-Perron test statistic. $\Delta$ is the first difference operator. P-values are Mackinnon (1996) one-sided p-values. Critical values are listed as well.

Table 3: Results of Univariate Unit Root tests on the spot dollar real exchange rates 
Table 4: Results of the Linearity Tests

A. P-Values for delay parameter, $d$

\begin{tabular}{|c|c|c|c|c|c|}
\hline d & US-CAN & US-FRA & US-ITA & US-JAP & US-UK \\
\hline 1 & 0.820 & 0.666 & 0.090 & 0.829 & 0.025 \\
\hline 2 & 0.934 & 0.530 & 0.069 & 0.796 & 0.103 \\
\hline 3 & 0.936 & 0.469 & 0.246 & 0.621 & 0.127 \\
\hline 4 & 0.963 & 0.624 & 0.241 & 0.854 & 0.126 \\
\hline 5 & 0.891 & 0.418 & 0.306 & 0.922 & 0.059 \\
\hline 6 & 0.814 & 0.086 & 0.088 & 0.990 & 0.006 \\
\hline 7 & 0.794 & 0.138 & 0.061 & 0.974 & 0.004 \\
\hline 8 & 0.836 & 0.263 & 0.067 & 0.818 & 0.029 \\
\hline 9 & 0.910 & 0.236 & 0.044 & 0.939 & 0.035 \\
\hline 10 & 0.852 & 0.114 & 0.017 & 0.910 & 0.019 \\
\hline 11 & 0.556 & 0.068 & 0.019 & 0.793 & 0.239 \\
\hline 12 & 0.153 & 0.322 & 0.031 & 0.742 & 0.523 \\
\hline \multicolumn{6}{|c|}{ B. P-Values of the Hypothesis Tests } \\
\hline \multicolumn{6}{|l|}{ Test } \\
\hline Statistic & & US-FRA & US-ITA & US-UK & \\
\hline $\mathrm{p}$ & & 4 & 2 & 3 & \\
\hline d & & 11 & 10 & 7 & \\
\hline $\mathrm{H}_{0, \mathrm{LIN}}$ & & 0.068 & 0.017 & 0.004 & \\
\hline $\mathrm{H}_{0,1}$ & & 0.204 & 0.084 & 0.086 & \\
\hline $\mathrm{H}_{0,2}$ & & 0.043 & 0.052 & 0.001 & \\
\hline $\mathrm{H}_{0,3}$ & & 0.392 & 0.098 & 0.926 & \\
\hline
\end{tabular}

Table 5. Unrestricted ESTAR results.

\begin{tabular}{|c|c|c|c|c|c|c|}
\hline & \multicolumn{2}{|c|}{ US-FRA } & \multicolumn{2}{|c|}{ US-ITA } & \multicolumn{2}{|c|}{ US-UK } \\
\hline & Coefficient & P-value & Coefficient & P-value & Coefficient & P-value \\
\hline $\mathrm{k}$ & 0.001 & 0.804 & 0.001 & 0.829 & -0.001 & 0.737 \\
\hline$\lambda$ & 0.070 & 0.105 & 0.015 & 0.551 & 0.021 & 0.298 \\
\hline$\phi_{1}$ & 0.261 & 0.094 & 0.025 & 0.865 & 0.366 & 0.000 \\
\hline$\phi_{2}$ & -0.148 & 0.402 & & & -0.116 & 0.122 \\
\hline$\phi_{3}$ & -0.065 & 0.739 & & & & \\
\hline$k^{*}$ & 0.006 & 0.375 & 0.004 & 0.550 & 0.517 & 0.983 \\
\hline$\lambda^{*}$ & -0.131 & 0.002 & -0.072 & 0.021 & -3.287 & 0.983 \\
\hline$\phi_{1}{ }^{*}$ & 0.029 & 0.883 & 0.523 & 0.002 & -3.054 & 0.983 \\
\hline$\phi_{2}{ }^{*}$ & 0.058 & 0.795 & & & -5.095 & 0.983 \\
\hline$\phi_{3}{ }^{*}$ & 0.233 & 0.316 & & & & \\
\hline$\gamma$ & 0.959 & 0.122 & 0.528 & 0.148 & 0.005 & 0.983 \\
\hline $\mathrm{C}_{0}$ & -0.077 & 0.005 & -0.151 & 0.000 & 0.097 & 0.002 \\
\hline$p$ & 4 & & 2 & & 3 & \\
\hline$d$ & 11 & & 10 & & 7 & \\
\hline $\begin{array}{l}\text { Sum squared } \\
\text { Residuals }\end{array}$ & 0.178 & & 0.170 & & 0.212 & \\
\hline
\end{tabular}




\begin{tabular}{|c|c|c|c|c|}
\hline \multirow[b]{2}{*}{ Variable } & \multicolumn{2}{|c|}{ US-UK } & \multicolumn{2}{|c|}{ US-Italy } \\
\hline & Parameter Estimate & Significance & Parameter Estimate & Significance \\
\hline$\phi_{0}$ & 0.003 & 0.16 & 0.001 & 0.46 \\
\hline $\mathrm{y}_{\mathrm{t}-1}$ & 1.301 & 0.00 & 1.321 & 0.00 \\
\hline$y_{t-2}$ & -0.406 & 0.00 & -0.336 & 0.00 \\
\hline$y_{t-3}$ & 0.091 & 0.058 & & \\
\hline Dummy(1979:07) & 0.09 & 0.00 & & \\
\hline Dummy(1985:04) & 0.103 & 0.00 & & \\
\hline Dummy(1992: 10) & -0.096 & 0.00 & & \\
\hline Dummy(1982:06) & & & -0.073 & 0.002 \\
\hline Dummy(1991:03) & & & -0.076 & 0.001 \\
\hline Dummy(1992: 10) & & & -0.083 & 0.001 \\
\hline$\overline{R^{2}}$ & 0.971 & & 0.97 & \\
\hline Adjusted $\mathrm{R}^{2}$ & 0.971 & & 0.97 & \\
\hline SSR & 0.2 & & 0.16 & \\
\hline Range & $1973: 1$ to $2005: 10$ & & $1973: 3$ to $1998: 12$ & \\
\hline Degrees of Freedom & 384 & & 30 & \\
\hline
\end{tabular}

Table 6: Re-estimation of Linear model with dummies

\begin{tabular}{lccc} 
No. of Trials & $\begin{array}{c}\text { Probability } \\
\text { of Rejection }\end{array}$ & $\operatorname{Pr}($ ESTAR|Rejection) & $\operatorname{Pr}($ LSTAR|Rejection) \\
\hline 100 & 0.350 & 0.457 & 0.543 \\
500 & 0.350 & 0.423 & 0.557 \\
1000 & 0.369 & 0.439 & 0.561
\end{tabular}

Table 7: Results from simulation 


\begin{tabular}{lcc}
\multicolumn{3}{c}{ A. P-Values for delay parameter, $d$} \\
\hline \hline$d$ & US-ITA & US-UK \\
\hline 1 & 0.815 & 0.757 \\
2 & 0.753 & 0.814 \\
3 & 0.798 & 0.781 \\
4 & 0.727 & 0.146 \\
5 & 0.512 & 0.396 \\
6 & 0.071 & 0.146 \\
7 & 0.119 & 0.077 \\
8 & 0.314 & 0.045 \\
9 & 0.246 & 0.112 \\
10 & 0.102 & 0.033 \\
11 & 0.211 & 0.148 \\
12 & 0.296 & 0.249 \\
\hline \hline
\end{tabular}

\begin{tabular}{lcc}
\multicolumn{3}{l}{ B. P-Values of the Hypothesis Tests } \\
\hline \hline \multicolumn{3}{l}{ Test } \\
Statistic & US-ITA \\
\hline $\mathrm{p}$ & 2 & 3 \\
$\mathrm{~d}$ & 6 & 10 \\
$\mathrm{H}_{0, \text { LIN }}$ & 0.071 & 0.0332 \\
$\mathrm{H}_{0,1}$ & 0.029 & 0.0091 \\
$\mathrm{H}_{0,2}$ & 0.489 & 0.3349 \\
$\mathrm{H}_{0,3}$ & 0.219 & 0.3596 \\
\end{tabular}

Table 8: Results from Linearity Tests without Influential Observations

\begin{tabular}{|c|c|c|c|c|}
\hline \multirow[b]{2}{*}{ Variable } & \multicolumn{2}{|c|}{ UK } & \multicolumn{2}{|c|}{ Italy } \\
\hline & $\begin{array}{l}\text { Parameter } \\
\text { Estimate }\end{array}$ & Significance & $\begin{array}{c}\text { Parameter } \\
\text { Estimate }\end{array}$ & Significance \\
\hline$D^{*} y_{t-1}$ & 1.561 & 0.000 & 1.309 & 0.000 \\
\hline$D^{*} y_{t-2}$ & -0.626 & 0.000 & -0.706 & 0.000 \\
\hline$(1-D) * y_{t-1}$ & 1.247 & 0.000 & 0.253 & 0.025 \\
\hline$(1-D) * y_{t-2}$ & -0.417 & 0.000 & 1.297 & 0.000 \\
\hline$(1-D) * y_{t-3}$ & 0.168 & 0.000 & -0.305 & 0.000 \\
\hline $\mathrm{R}^{2}$ & \multicolumn{2}{|c|}{0.97} & \multicolumn{2}{|c|}{0.975} \\
\hline Adjusted $\mathrm{R}^{2}$ & \multicolumn{2}{|c|}{0.97} & \multicolumn{2}{|c|}{0.974} \\
\hline SSR & \multicolumn{2}{|c|}{0.209} & \multicolumn{2}{|c|}{0.1676} \\
\hline Range & \multicolumn{2}{|c|}{$1973: 3$ to $2005: 9$} & \multicolumn{2}{|c|}{$1973: 1$ to $1998: 12$} \\
\hline Degrees of Freedom & \multicolumn{2}{|c|}{384} & \multicolumn{2}{|c|}{304} \\
\hline
\end{tabular}

Table 9: TAR Estimation Results 